\title{
AVALIAÇÃO DA VARREDURA LASER TERRESTRE EM DIFERENTES DISTÂNCIAS DA ÁRVORE PARA MENSURAR VARIÁVEIS DENDROMÉTRICAS
}

\author{
Evaluation of terrestrial laser scanning at different distances from the tree for \\ measuring dendrometric variables

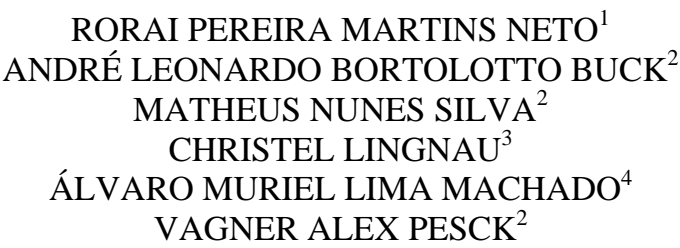 \\ ${ }^{1}$ Graduando em Engenharia Florestal, UFPR \\ ${ }^{2}$ Programa de Pós-Graduação em Engenharia Florestal, UFPR \\ ${ }^{3}$ Departamento de Ciências Florestais, UFPR, \\ Av. Prefeito Lothário Meissner, 900 \\ 80210-170, Jardim Botânico, Curitiba, PR, Brasil \\ ${ }^{4}$ Departamento de Geomática, UFPR \\ Rua: Cel. Francisco H dos Santos s/n, \\ 81531-990, Jardim das Américas, Curitiba, PR, Brasil \\ rorai.neto@gmail.com, andrenado@ibest.com.br, matheusnssilva@gmail.com, \\ lingnau@ufpr.br, alvaroml@ufpr.br, vagnerapesck@hotmail.com
}

\section{RESUMO}

O laser scanner terrestre é uma alternativa para a coleta de dados dendrométricos em campo sem a necessidade da derrubada da árvore. Assim, este estudo teve como objetivo avaliar a influência da distância da varredura laser terrestre na determinação das variáveis dendrométricas. Foram analisadas duas árvores com altura total de 17,14 m e 16,00 m. A varredura laser foi realizada a 5, 10, 15 e $20 \mathrm{~m}$ de distância da árvore. Os diâmetros foram obtidos em alturas parciais até o topo da árvore. Os resultados obtidos com a varredura laser foram validados com as medidas obtidas tradicionalmente em campo com suta e trena. A melhor distância para a obtenção das variáveis dendrométricas foi de $15 \mathrm{~m}$ para ambas as árvores. As 
distâncias menores que $10 \mathrm{~m}$ não foram indicadas para a mensuração das duas árvores analisadas. A determinação da altura total foi coerente com as medidas paramétricas. Foi possível a determinação de diâmetros até 55\% da altura total e, acima, a ramificação da árvore resultou em uma super ou subestimativa. A melhor distância de varredura possivelmente está atrelada à altura da árvore.

Palavras-chave: Laser Scanner Terrestre; Diâmetros; Distância de Varredura; Alturas Relativas; Modelagem do Tronco.

ABSTRACT
The terrestrial laser scanner is presented as an alternative to dendrometric data collection in the field without the necessity of cutting down trees. This study aimed at the evaluation of the scanning distance for dendrometric variables determination. Two trees have been used in the study, with total height of 17.14 and $16.00 \mathrm{~m}$. Data collection has been made at distances of 5, 10, 15 and $20 \mathrm{~m}$. Diameters have been obtained in partial heights up to the top of the tree. Data has been validated by using the caliper and tape. The best scanning distance to obtain reliable variables has been at $15 \mathrm{~m}$ for both trees. Distances closer than $10 \mathrm{~m}$ are not recommended in this case. Total height determination has been coherent with the parametric measurements. Diameter estimative has been possible up to $55 \%$ of tree height and above that, resulted in over or under-estimative. The best scanning distance is possibly linked to the tree height.

Keywords: Terrestrial Laser Scanning; Diameters; Scanning Distances; Relative Height, Stem Modeling.

\section{INTRODUÇÃO}

O inventário e o manejo florestal requerem a medição de parâmetros que descrevam a geometria das árvores, e, convencionalmente, esses parâmetros se limitam à altura e ao DAP (diâmetro à altura do peito, medido a 1,30 m da base da árvore). Em alguns casos, outros parâmetros são necessários, como diâmetros em diferentes alturas para a cubagem da árvore, os quais são impossíveis de obter sem a derrubada da árvore (BIENERT et al., 2006a).

O laser scanner terrestre é uma ferramenta interessante na área florestal, pois, por meio da varredura da superfície do tronco, é possível reconstituir a sua forma orgânica com alta precisão e rapidez. A reconstituição da forma orgânica do tronco se dá por meio do processamento da nuvem de pontos tridimensional, a qual permite a modelagem e determinação de variáveis dendrométricas, como diâmetros em alturas parciais, altura total e volume, sem a derrubada da árvore (BIENERT et al., 2006a; MAAS et al., 2008).

A nuvem de pontos pode ser analisada em ambiente computacional, minimizando erros causados por condições adversas em campo, como vegetação densa, desacertos na leitura das medidas ou até cansaço da equipe (HOPKINSON et al., 2004). 
Thies e Spiecker (2004) encontraram erros de 1,3\% na medição do DAP quando foram comparados os resultados obtidos por meio da varredura laser e a medição convencional em campo. Da mesma forma, em um estudo semelhante realizado por Hopkinson et al. (2004), foi encontrado um coeficiente de correlação de 0,92 entre as estimativas convencionais e as do laser para o DAP e a altura total.

A volumetria do tronco de uma árvore pode ser obtida por meio do modelo tridimensional com dados da varredura laser. Buck et al. (2012) obtiveram resultados muito próximos ao valor paramétrico de determinação de volumes. Este valor paramétrico pode ser alcançado utilizando o método do deslocamento de água. O instrumento utilizado para este fim é designado na Engenharia Florestal como “Xilômetro”. O princípio do método pode ser entendido de acordo com o seguinte raciocínio: em um recipiente onde o volume inicial de água (pré-imersão da tora) é estabelecido e determinado por certo nível, é possível verificar a mudança de altura da água na escala graduada (pós-imersão da tora) e obter o volume pela quantidade de água deslocada.

Estudos que visam à determinação de variáveis dendrométricas levando em consideração a melhor posição do equipamento laser e a distância de varredura, de acordo com as particularidades do ambiente florestal, são fundamentais para o planejamento da coleta de dados. Todavia poucos trabalhos tratam essa temática. Litkey et al. (2008) realizaram um estudo avaliando a distância de varredura, sendo que o enfoque foi a detecção automática de árvores. As distâncias de até $15 \mathrm{~m}$ permitiram a identificação correta de $100 \%$ das árvores para uma floresta de coníferas na Finlândia.

Assim, o presente estudo tem como objetivo avaliar a influência da distância de posicionamento do equipamento para obtenção de variáveis dendrométricas, diâmetros em diferentes alturas e altura total, através dos dados laser scanner terrestre. Os resultados serão validados por meio de comparações com medidas tradicionais em campo com a suta e a trena, para os diâmetros em diferentes alturas e a altura total, respectivamente.

\section{MATERIAIS E MÉTODOS}

\subsection{Coleta de Dados}

O estudo foi realizado em duas árvores de um povoamento inequiâneo de Pinus spp., com uma densidade de 1.275 árvores.ha $^{-1}$, sem tratamentos silviculturais (poda e desbaste) e com uma idade média de 12 anos.

A coleta de dados consistiu em duas etapas: varredura laser com as árvores em pé, a qual foi realizada com o equipamento Leica Geosystems HDS3000 (Figura 1 e Tabela 1) e medições de altura e diâmetros com as árvores derrubadas, utilizando suta e trena.

Para a determinação das variáveis dendrométricas (diâmetros e altura total) a partir do uso da tecnologia laser, foram realizadas varreduras simples em diferentes distâncias de cada árvore: 5, 10, 15 e 20 m, com uma resolução de $1 \mathrm{~cm}$ na 
horizontal e $2 \mathrm{~cm}$ na vertical em relação ao plano horizontal estabelecido pelo eixo da árvore em pé. As resoluções foram mantidas em todas as diferentes distâncias, para fins de comparação, alterando-se apenas o parâmetro range (garante a resolução especificada para diferentes distâncias).

Figura 1 - Varredura laser terrestre no povoamento de Pinus spp.

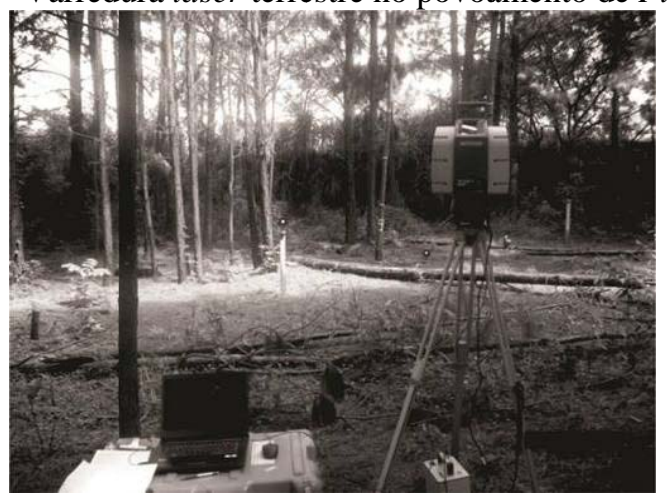

Tabela 1 - Especificações técnicas do equipamento Leica HDS3000 (Fonte: Leica

\begin{tabular}{l|l}
\multicolumn{2}{c}{ Geosystems). } \\
\hline Características técnicas & Até 4000 pontos por segundo \\
\hline Aquisição de pontos & $360^{\circ}$ (horizontal) x $270^{\circ}$ (vertical) \\
Campo de visão & $6 \mathrm{~mm} / 4 \mathrm{~mm}(1$ a $50 \mathrm{~m})-1$ sigma \\
Acurácia posição / distância & 60 microrad x 60 microrad - 1 sigma \\
IFOV & Até $100 \mathrm{~m}$ \\
Alcance &
\end{tabular}

Os diâmetros foram obtidos em alturas relativas de 0, 5, 10, 15, 25, 35, 45, 50 e $55 \%$, e acima, a cada $10 \%$, até o topo da árvore (Figura 2). Com a árvore derrubada, foram obtidos os diâmetros supracitados, utilizando-se suta, e a altura total, mensurada com trena.

Figura 2 - Obtenção de diâmetros em alturas relativas.

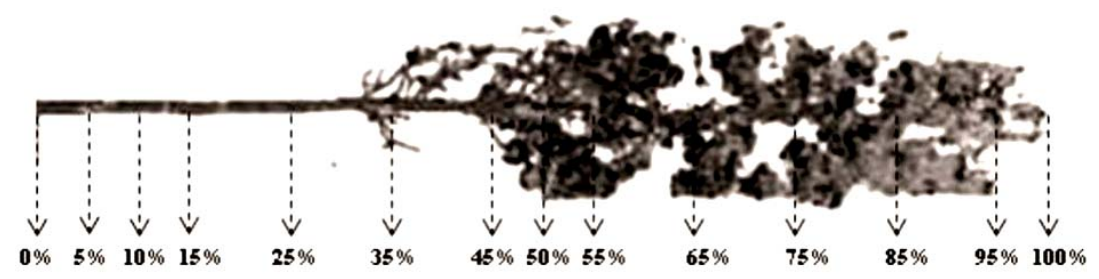

Bol. Ciênc. Geod., sec. Artigos, Curitiba, v. 19, no 3, p.420-433, jul-set, 2013. 


\subsection{Processamento de Dados Laser}

O processamento da nuvem de pontos gerada pela varredura laser consistiu em três etapas: isolamento da árvore, filtragem e determinação das variáveis dendrométricas.

O isolamento da árvore visou obter uma nuvem de pontos correspondente ao objeto de interesse. A filtragem teve a finalidade de eliminar pontos não pertencentes ao tronco, como galhos e sub-bosque. Ambos os processos foram realizados de forma manual, com ferramentas de edição em software específico. A determinação das alturas e dos diâmetros das árvores foram obtidos por meio de algoritmos implementados em ambientes de programação.

\subsubsection{Determinação de Alturas}

A altura total foi determinada por dois métodos. O primeiro método, denominado de diferença de cotas, determina a altura da árvore através da subtração entre as coordenadas $\mathrm{Z}$ dos pontos de maior e de menor cota oriundos da filtragem manual (SILVA et al., 2011). O segundo método, denominado de média das cotas, adota a coordenada $\mathrm{Z}$ do ponto de menor cota, obtém uma média dos 20 pontos de maior cota e determina a altura da árvore pela subtração dessas duas cotas. De acordo com Lingnau et al. (2009), a determinação precisa da altura total está ligada aos processos de filtragem realizados anteriormente, ou seja, um método não eficiente de filtragem faz com que ocorram erros tanto na determinação da base da árvore quanto na determinação do topo da copa da árvore.

\subsubsection{Determinação de Diâmetros}

Os diâmetros foram calculados por dois métodos diferentes: o método da distância máxima e o método da circunferência ótima.

O método da distância máxima (SILVA, 2011) consiste na leitura dos dados da seção transversal e determinação das coordenadas $X_{\min }, X_{\max }, Y_{\min }$ e $Y_{\max }$, correspondentes a mínimas e máximas das abscissas e ordenadas a uma dada altura da árvore e com uma espessura " $n$ " (Figura 3). A espessura da seção transversal adotada neste estudo foi de $1 \mathrm{~cm}$, a qual estabelece uma quantidade de pontos considerável para o cálculo das distâncias nas abscissas e ordenadas. A maior distância planimétrica entre as distâncias " $X_{\max }-X_{\min }$ " e " $Y_{\max }-Y_{\min }$ " é escolhida como primeira aproximação do diâmetro. A seguir, avalia-se a distância de todos os pontos da seção analisada com relação a esses dois pontos, substituindo-se a distância tomada como referência sempre que uma distância maior for encontrada. Ao final, a distância entre os pontos mais afastados é adotada como diâmetro da seção transversal (Figura 3).

O método da circunferência ótima ajusta uma circunferência à nuvem de pontos de uma seção transversal por meio da minimização do desvio padrão da distância euclidiana entre um centro hipotético (centro de massa) e os pontos que definem a seção transversal em determinada altura do tronco, utilizando o método Simplex (SILVA, 2011) (Figura 4).

Bol. Ciênc. Geod., sec. Artigos, Curitiba, v. 19, nº 3, p.420-433, jul-set, 2013. 
Figura 3 - Método da distância máxima.

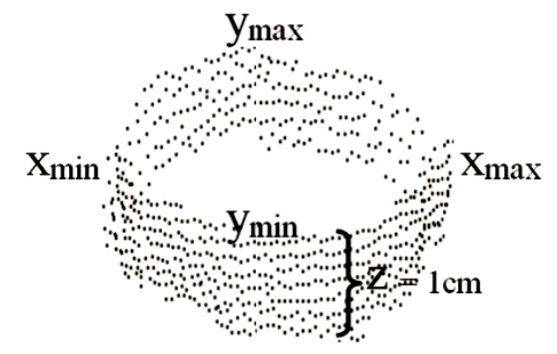

O ponto central ótimo, que minimiza essa função, é o centro da circunferência ajustada. A distância média entre o ponto central ótimo e os demais pontos é considerado o raio da seção transversal, a partir do qual é determinado o diâmetro (ASCHOFF et al., 2004; LIANG et al., 2008; BIERNET et al., 2006b; BIERNET et al., 2007; LITKEY et al., 2008; SILVA, 2011).

Figura 4 - Método da circunferência ótima.

Fonte: BUCK, 2012 (Modificado).
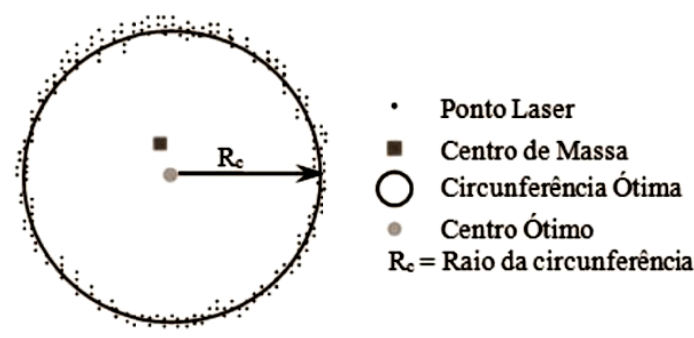

\section{RESULTADOS E DISCUSSÕES}

\subsection{Processamento de Dados Laser}

As árvores isoladas da nuvem de pontos e filtradas (Figura 5) apresentam uma copa grande e muito densa, ou seja, o fuste, nessa porção, ficou totalmente encoberto pelas acículas, não permitindo sua modelagem. Assim, foi possível filtrar somente até $50 \%$ da altura total, na árvore 1 , e 35\%, na árvore 2. A varredura em diferentes distâncias não interferiu na melhoria da visibilidade do fuste na região da copa.

\subsubsection{Determinação de Alturas}

Para fins de validação, as alturas totais das árvores 1 e 2 foram medidas com a trena, sendo obtidos os valores 17,14 m e 16,00 m, respectivamente. A distância 
mais indicada para o posicionamento do equipamento para as duas árvores foi de 15 $\mathrm{m}$, na qual foram observados os menores erros em altura (-0,53\% para a árvore 1 e 3,67\% para a árvore 2). A distância de $5 \mathrm{~m}$ foi a menos indicada para ambas as árvores, visto que nessa distância foram obtidos os maiores erros (6,15\% para a árvore 1 e 10,04\% para a árvore 2). Nesta distância, devido ao ângulo de visada na vertical ser mais inclinado, o pulso laser capta muitos galhos e acículas, impedindo a visualização fidedigna do fuste. A determinação da altura total da árvore apresentou o melhor resultado por meio do método da média das cotas, quando comparado com todas as distâncias de varredura analisadas (Figura 6).

Figura 5 - Imagem das árvores isoladas e filtradas geradas pela varredura laser.

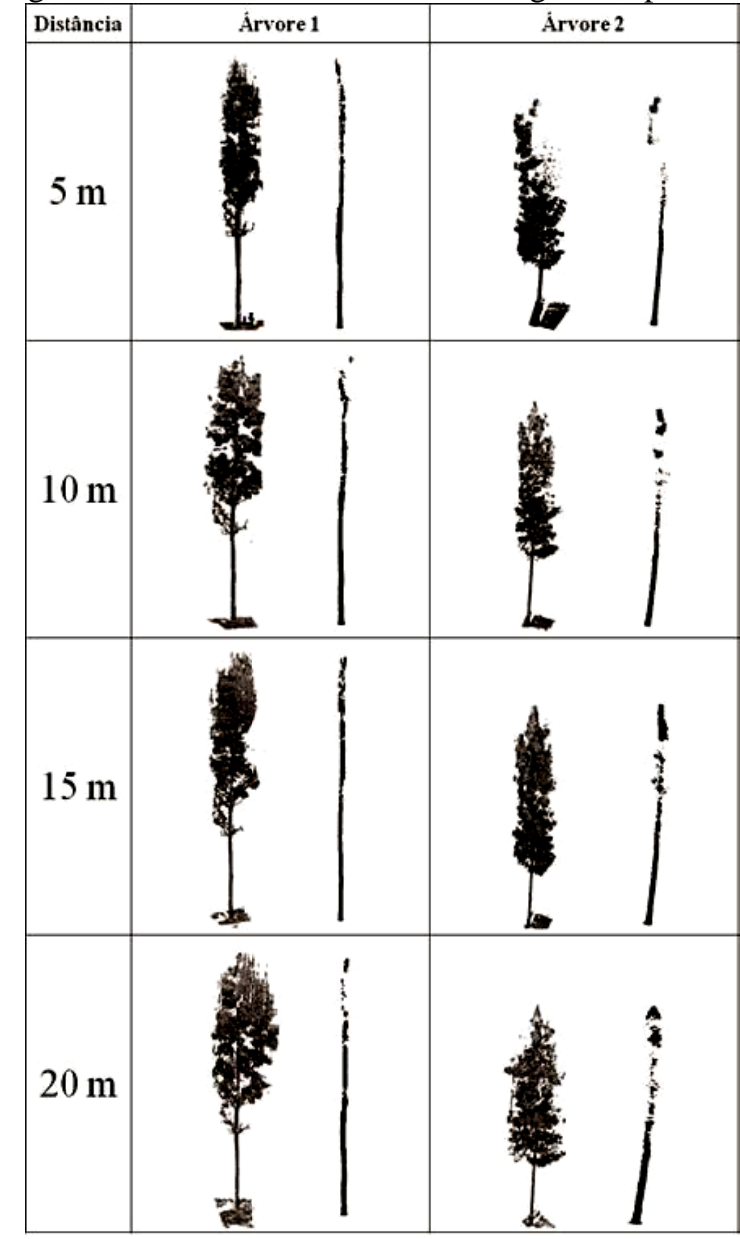

Bol. Ciênc. Geod., sec. Artigos, Curitiba, v. 19, no 3, p.420-433, jul-set, 2013. 
A determinação da altura usando o método da média das cotas foi adequada, porém, erros maiores foram observados na árvore 2, devido à intervenção de copas adjacentes no campo de visada do laser. As super e subestimativas das alturas foram igualmente observadas no processamento dos dados laser por outros autores (LINGNAU et al., 2009; HOPKINSON et al., 2004), os quais atribuem os erros à alta densidade de copa, ao posicionamento do equipamento e a uma baixa densidade amostral de pontos no dossel superior.

Figura 6 - Altura da árvore obtida pelos métodos diferença e média das cotas a diferentes distâncias de varredura.

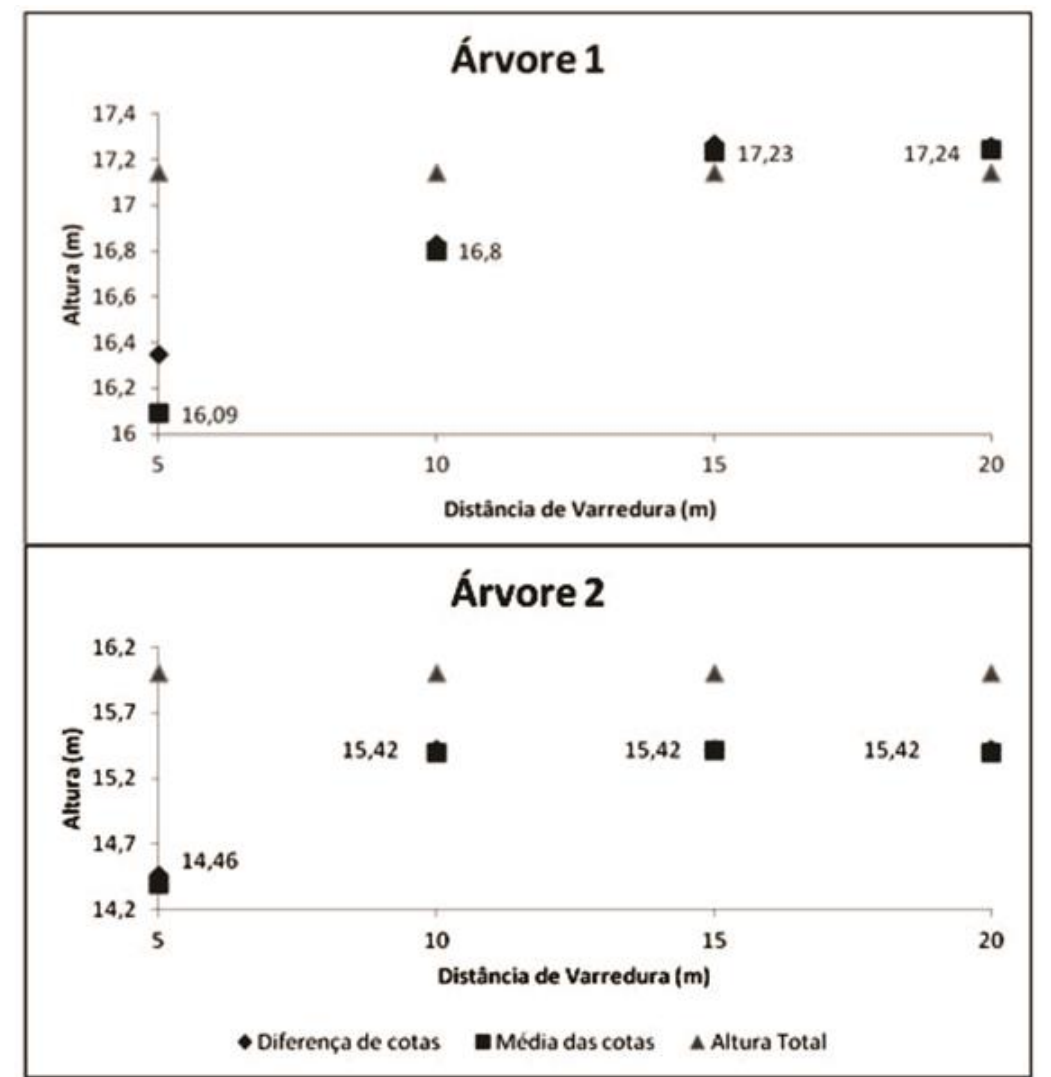

\subsubsection{Determinação de Diâmetros}

Os diâmetros nas alturas relativas (Figura 2) foram obtidos a partir da modelagem tridimensional da nuvem de pontos, por meio dos métodos da distância máxima e circunferência ótima. Os resultados foram comparados com os diâmetros 
mensurados com a suta (Figura 7). A distância de varredura da árvore que apresentou os melhores resultados foi a de $15 \mathrm{~m}$.

Neste estudo, as distâncias menos indicadas para a mensuração de árvores encontram-se abaixo de $10 \mathrm{~m}$, sendo que a árvore 1 apresentou o maior erro com o método da distância máxima à distância de $10 \mathrm{~m}$, e a árvore 2, com o método da circunferência ótima à distância de $5 \mathrm{~m}$. Esse erro pode ser atribuído à problemática de que, em distâncias menores, o ângulo de visada é mais inclinado e assim a varredura contempla muitos galhos sobrepostos, os quais acabam por dificultar a aquisição de dados na superfície do tronco nas partes mais altas da árvore. Assim, a partir de distâncias mais próximas à árvore, como, por exemplo, 5 e 10 metros, a modelagem do fuste mostrou-se inconstante, sendo possível a obtenção de diâmetros somente até uma média de 35\% da altura total (Figura 8).

Figura 7 - Modelagem do tronco obtido por meio da varredura laser terrestre a 15 m de distância.

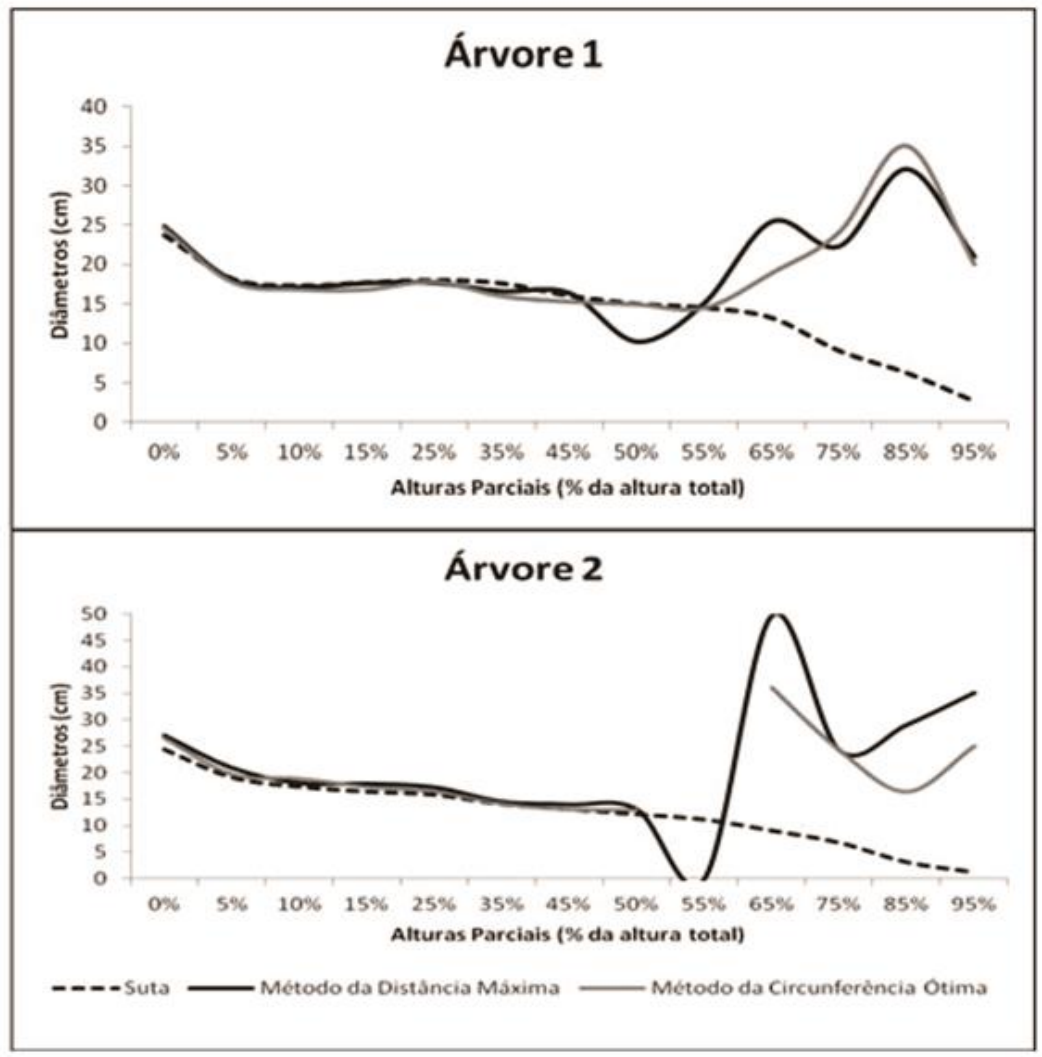

Bol. Ciênc. Geod., sec. Artigos, Curitiba, v. 19, no 3, p.420-433, jul-set, 2013. 
Figura 8 - Modelagem do fuste com dados da varredura laser para as distâncias de 10 e 5 m, respectivamente, para as árvores 1 e 2.

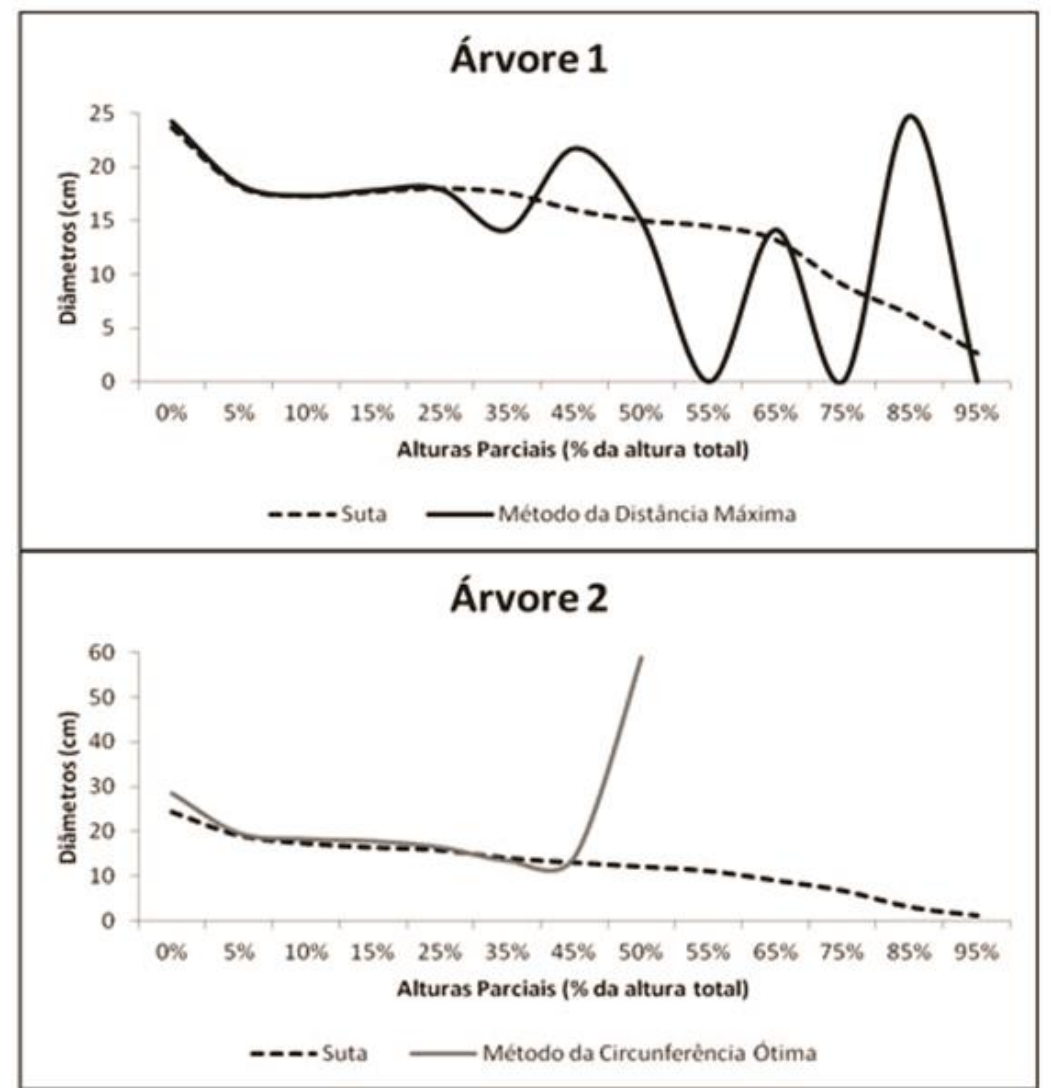

A determinação de diâmetros foi possível apenas até 55\% da altura total da árvore, devido à alta ramificação na região das copas quando comparadas com a medição usando suta. Gatziolis et al. (2010) relataram que podem ser encontrados obstáculos na medição de diâmetros na parte superior das árvores, em função de suas copas. Por esse motivo, sugerem a interpolação para a estimativa dos diâmetros em porções do fuste onde não podem ser determinados, pela pequena quantidade de pontos.

Os resultados obtidos na determinação dos diâmetros a uma distância de varredura de $15 \mathrm{~m}$ da árvore para a porção de 55\% da altura total, com os métodos da distância máxima e da circunferência ótima, quando comparados com a medição com suta, apresentaram uma distribuição de resíduos similares para ambas as árvores (Figura 9). 
A distribuição dos resíduos para ambas as árvores (Figura 9) indica que os melhores resultados são obtidos nas porções com maiores diâmetros, ou seja, as partes mais baixas da árvore, as quais estão livres de galhos e copa.

Figura 9 - Distribuição de resíduos para as árvores 1 e 2 referente à distância de varredura de $15 \mathrm{~m}$.

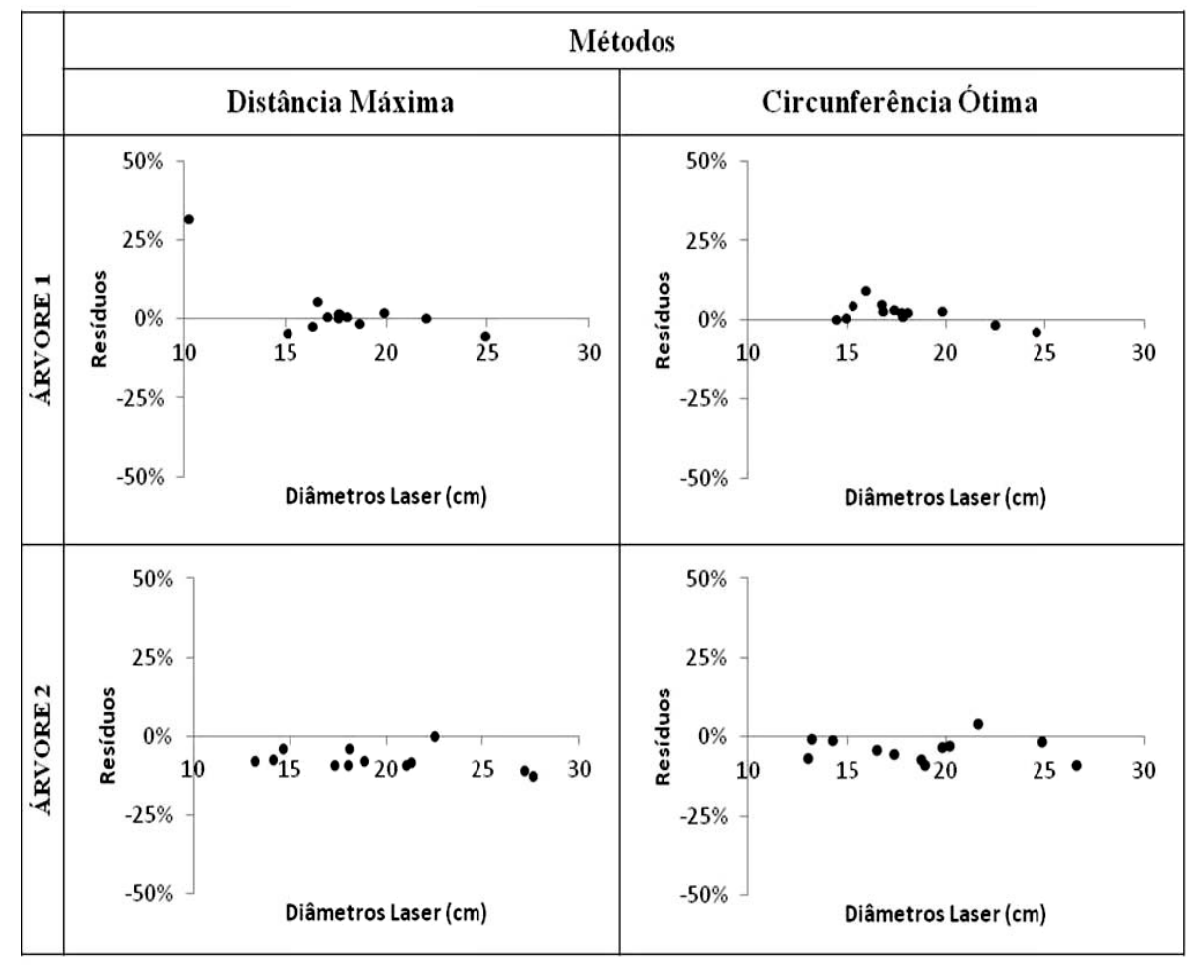

A Tabela 2 mostra as estatísticas dos resíduos com a média dos erros e o desvio padrão em porcentagem dos diâmetros obtidos até 55\% da altura total, com os métodos da distância máxima e circunferência, para as distâncias de varredura de 5, 10, 15 e $20 \mathrm{~m}$.

É possível verificar pelas estatísticas (Tabela 2) que a distância de $15 \mathrm{~m}$ foi a que teve os menores erros médios e desvios padrões para ambas as árvores com os dois diferentes métodos, comprovando assim que essa distância de varredura foi a mais indicada no estudo. Porém, na árvore 2, nota-se uma maior discrepância entre os dados, pelo fato de haver a copa de outra árvore adjacente à copa da árvore 2, ocasionado assim maiores erros. 
Tabela 2 - Estatísticas dos resíduos dos diâmetros para as duas árvores, nas diferentes distâncias de varredura.

\begin{tabular}{|c|c|c|c|c|}
\hline Árvore & Método & Distância (m) & $\begin{array}{c}\text { Média dos } \\
\text { erros (\%) }\end{array}$ & $\begin{array}{c}\text { Desvio padrão } \\
(\%)\end{array}$ \\
\hline \multirow{8}{*}{1} & \multirow{4}{*}{ DM } & 5 & 7,43 & 31,53 \\
\hline & & 10 & 6,24 & 30,56 \\
\hline & & 15 & 2,70 & 9,29 \\
\hline & & 20 & 5,85 & 29,10 \\
\hline & \multirow{4}{*}{$\mathrm{CO}$} & 5 & 3,42 & 18,51 \\
\hline & & 10 & 8,11 & 29,60 \\
\hline & & 15 & 2,37 & 3,22 \\
\hline & & 20 & 6,01 & 29,04 \\
\hline \multirow{8}{*}{2} & \multirow{4}{*}{$\mathrm{DM}$} & 5 & 1,07 & 34,03 \\
\hline & & 10 & 14,43 & 38,17 \\
\hline & & 15 & 0,87 & 29,97 \\
\hline & & 20 & 12,17 & 38,83 \\
\hline & \multirow{4}{*}{$\mathrm{CO}$} & 5 & $-25,26$ & 111,26 \\
\hline & & 10 & 6,93 & 44,89 \\
\hline & & 15 & 4,22 & 29,01 \\
\hline & & 20 & 10,36 & 41,06 \\
\hline
\end{tabular}

DM = distância máxima; $\mathrm{CO}$ = circunferência ótima.

\section{CONCLUSÃO}

O estudo em questão mostrou que a melhor distância de posicionamento do equipamento para a obtenção tanto de diâmetros ao longo do tronco quanto de altura total foi de $15 \mathrm{~m}$. Essa distância, muito provavelmente, está atrelada à altura da árvore, pois neste estudo as duas árvores analisadas possuem em média 16,6 m, e os melhores resultados foram observados na varredura com distância de $15 \mathrm{~m}$.

A distância de $5 \mathrm{~m}$ foi a que se mostrou menos adequada para determinação da altura total, pois, devido à proximidade da árvore, o ângulo de visada do laser na vertical impede a varredura da superfície do tronco. Nesse caso, a intervenção de copas adjacentes também contribuiu para aumentar os erros.

Na determinação dos diâmetros, as distâncias de 5 e 10 m não apresentaram bons resultados. Nesse caso, a ausência de tratos silviculturais (poda e desbaste) foi um fator que impediu a obtenção de diâmetros acima de 55\% da altura total, sub ou superestimando-os. O método da circunferência ótima mostrou os menores desvios para a distância de $15 \mathrm{~m}$.

Outros estudos devem ser realizados para a obtenção de variáveis dendrométricas a diferentes distâncias em povoamentos florestais, em que a densidade de árvores.ha ${ }^{-1}$ e o relevo devem ser considerados.

\section{AGRADECIMENTOS}

Ao Departamento de Geomática da Universidade Federal do Paraná, pelo empréstimo do equipamento Laser HDS3000, que contribuiu para a realização deste trabalho. 


\section{REFERÊNCIAS BIBLIOGRÁFICAS}

ASCHOFF, T.; SPIECKER, H. Algorithms for the automatic detection of trees in laser scanner data. International Archives of Photogrammetry, Remote Sensing and Spatial Information Sciences, v. xxxvi, 8/w2. Freiburg, 2004.

BIENERT, A.; MAAS, H. G.; SCHELLER, S. Analysis of the information content of terrestrial laser scanner point clouds for the automatic determination of forest inventory parameters. In: Workshop on 3D Remote Sensing in Forest, 14-15. Vienna, 2006a.

BIENERT, A., SCHELlER, S., KEANE, E., MULLOOLY, G.; MOHAN, F. Application of terrestrial laser scanners for the determination of forest inventory parameters. International Archives of Photogrammetry, Remote Sensing and Spatial Information Sciences, 36. 2006b.

BIENERT, A. et al. Tree detection and diameter estimations by analysis of forest terrestrial laser scanner point clouds. ISPRS Workshop on Laser Scanning 2007 and SilviLaser 2007. Finland, 2007.

BUCK, A. L. B ; SILVA, M. N. ; LINGNAU, C. ; Machado, A.M.L ; Martins Neto, R. P. ; Pesck, V. A. . Avaliação da modelagem tridimensional do tronco de Pinus elliottii a partir de dados laser terrestre. Ambiência. v. 8, p. 549-557, 2012.

BUCK, A. L. B. Análise de métodos de filtragemem dados laser scanner terrestre aplicados à modelagem tridimensional do tronco de Pinus spp. 2012. 130f. Dissertação (Mestrado em Engenharia Florestal) - Universidade Federal do Paraná, UFPR, Curitiba, 2012.

GATZIOLIS, D.; POPESCU, S.; SHERIDAN, R.; KU, N. Evaluation of terrestrial lidar technology for the development of local tree volume equations. In: SILVILASER $10^{\circ}$. Freiburg. Proceedings... Freiburg, 2010. 1 CD-ROM.

HOPKINSON, C.; CHASMER, L., YOUNG-POW, C., TREITZ, P., 2004. Assessing forest metrics with a ground-based scanning lidar. Canadian Journal of Forest Research, 34, p. 573-583.

LIANG, X.; LITKEY, P.; HYYPPA, J.; KUKKO, A.; KAARTINEN, H.; HOLOPAINEN, M.. Plot-level trunk detection and reconstruction using one scan mode terrestrial laser scanning data. 2008 International workshop on Earth Observation and Remote Sensing Applications, IEEE, China, Beijing, 30 June-2 Jul, 2008.

LINGNAU, C.; MACHADO, A. M. L.; BAVARESCO JÚNIOR, A.; COLLA, F. L. Determinação de diâmetros a diferentes alturas de árvores individuais a partir de dados laser terrestre. In: Simpósio Brasileiro de Sensoriamento Remoto, 14, 2009, Natal. Anais... Natal: INPE, 2009.

LITKEY, P.; LIANG, X.; HYYPPÄ, J.; KUKKO, A.; KAARTINEN, H.; HOLOPAINEN, M.. Single-scan TLS methods for forest parameter retrieval. In: Silvilaser 2008, 2008, Edinburgh UK. p. 295-304. 
MAAS, H.-G.; BIENERT, A.,SCHELLER, S.; KEANE, E. Automatic forest inventory parameter determination from terrestrial laser scanner data. International journal of remote sensing, v. 29, n. 5, p. 1579-1593, 2008.

SILVA, M. N.; BUCK, A. L. B.; LINGNAU, C.; MACHADO, A. M. L.; MIRANDA, B. P. Varredura laser terrestre para determinação das variáveis dendrométricas da Araucaria angustifolia. In: Simpósio Brasileiro de Sensoriamento Remoto, 15, 2011, Curitiba. Anais... Curitiba: INPE, 2011.

SILVA, M. N. Aplicação de laser scanner terrestre para determinação de variáveis dendrométricas em Pinus spp. 2011. 126 f. Dissertação (Mestrado) - Curso de Engenharia Florestal, Departamento de Ciências Florestais, UFPR, Curitiba, 2011.

THIES, M.; SPIECKER, H. Evaluation and future prospects of terrestrial laserscanning for standardized forest inventories. International Archives of Photogrammetry, Remote Sensing and Spatial Information Sciences, v. xxxvi, 8/w2. Freiburg, 2004.

(Recebido em abril de 2012. Aceito em junho de 2013). 